\title{
Assessment of boundary-element method for modelling a free-floating sloped wave energy device. Part 2: experimental validation.
}

\author{
Grégory S. Payne* , Jamie R.M. Taylor, Tom Bruce, \\ Penny Parkin
}

Institute for Energy Systems, School of Engineering and Electronics, University of Edinburgh, Edinburgh EH9 3JL, UK

\begin{abstract}
Boundary element method has been widely used as a design tool in the offshore and ship building industry for more than 30 years. Its application to wave energy conversion is however more recent. This is the second of two papers on a comparison of numerical and physical modelling of a free-floating sloped wave energy converter. In the first paper the numerical modelling formulation for the power take-off mechanism was derived using the boundary-element method package WAMIT. It was verified against numerical benchmark data. In this paper, the outcome of the modelling of the whole device is compared with experimental measurements obtained from model testing in a wave tank. The agreement is generally good.
\end{abstract}

Key words:

wave energy, numerical modelling, experimental validation, WAMIT, physical modelling 


\section{Introduction}

The sloped wave energy converter concept considered was originally invented by Stephen Salter (Salter and Lin, 1995). It consists of a floating buoy with a power take-off mechanism that carries its own internal reaction mass in the form of a body of water contained within a large incline tube which is open to the sea. The tube, rigidly connected to the buoy, is blocked by a piston that can slide along its axis. As wave action induces motion of the buoy and the tube, the piston tends to stay relatively stationary due to the large added inertia of the water on each of its sides. There is therefore a relative motion between the piston and the buoy which can be exploited to produce energy.

In a previous companion paper the power take-off mechanism was modelled using the boundary-element method package WAMIT (WAMIT, Inc., 2000). The model was first derived for the case where the tube axis is vertical and then extended to the sloped configuration. Verification of the model was carried out against benchmark computations.

In the present paper, the whole wave energy converter is modelled and the modelling is validated against experimental measurement in regular waves. Two configurations of increasing complexity are considered.

(1) Power take-off mechanism disabled.

(2) Power take-off mechanism enabled.

The experimental data are obtained from model testing in the Edinburgh Curved Tank.

* Corresponding author.

Email address: gregory.payne@ed.ac.uk (Grégory S. Payne). 


\section{Experimental equipment}

\subsection{The Edinburgh Curved Tank}

Experimental measurements were carried out in the Edinburgh Curved Tank. It is a three dimensional wave tank with an array of 48 flap -type wave-makers configured in an arc. The arc radius is $9 \mathrm{~m}$ and its inclusive angle is just over $90^{\circ}$. Each wave-maker is about $305 \mathrm{~mm}$ wide. Water depth is $1.2 \mathrm{~m}$.

Due to space restriction, it is not possible to use a gently-sloped beach. The design adopted consists of triangular wedge-shaped cages arranged side-by-side (see figure 1). These cages are filled with a mixture of cut blocks of polyester, open cell foam and folded geotextile fabric sheets. The later is woven from small diameter PVC filaments. More detail on the Curved Tank can be found in (Taylor et al., 2003; Cruz et al., 2006).

\subsection{Experimental model}

The model consists of an inertia plate and a self-contained dynamometer tube acting as a power take-off. The components of the model are shown in figure 2.

The total length of the dynamometer tube is $1130 \mathrm{~mm}$. Its outside diameter is $120 \mathrm{~mm}$.

The buoy head is made of a close-cell PVC foam known as Devinycell. The grade used has a density of $100 \mathrm{~kg} \cdot \mathrm{m}^{-3}$ which provides good floatability. It also offers good mechanical and machining properties. Best surface finish is 


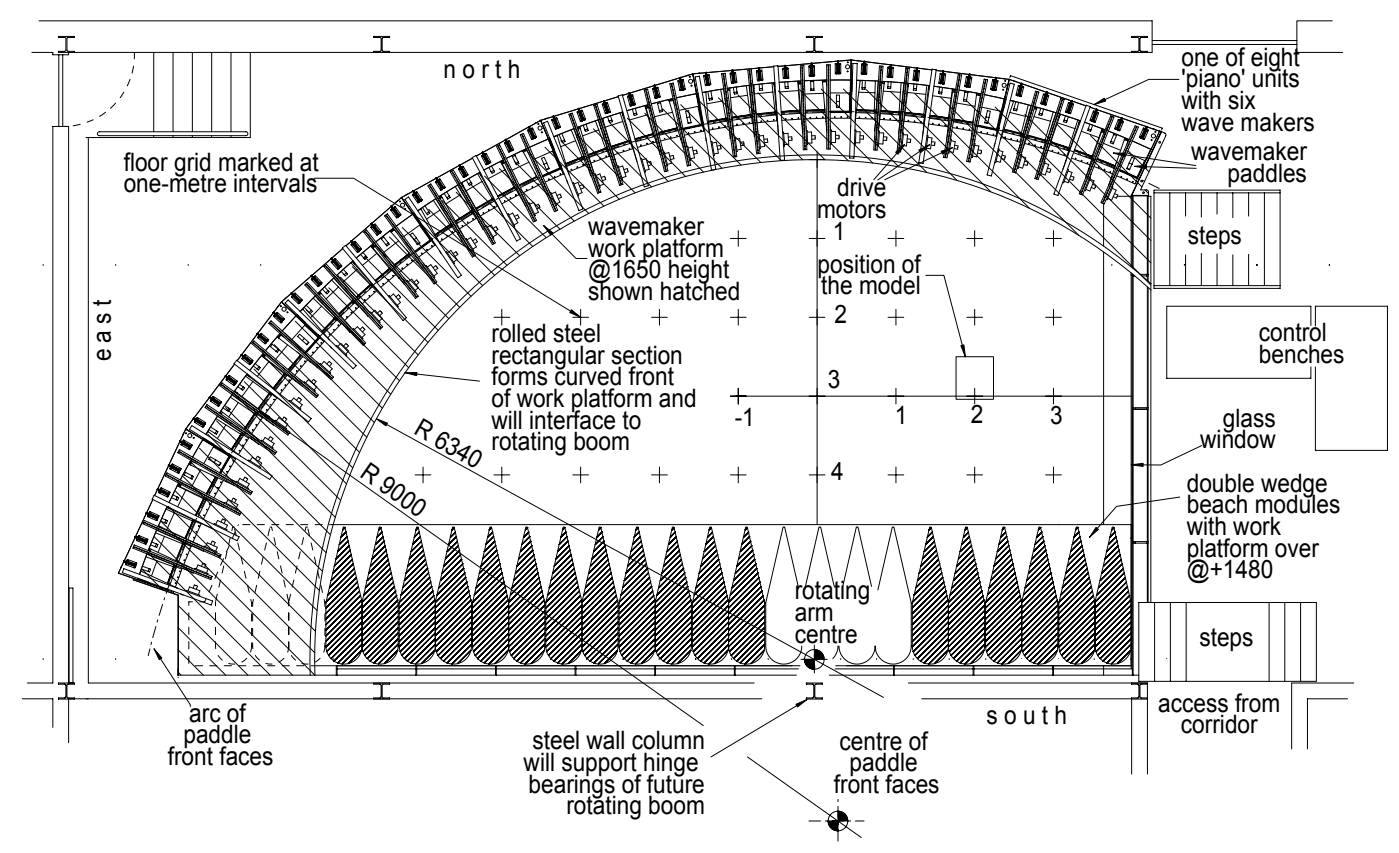

Fig. 1. Plan view of the Edinburgh Curved Tank.

achieved with sharp tools and an appropriate cutting speed. The advantage of the closed-cell feature of this foam is the low water absorption. Indeed, water tends to only penetrate a superficial external layer of the foam which corresponds to the cells burst open by cutting or machining processes.

The foam is sold in slabs with a maximum thickness of $50 \mathrm{~mm}$, so the buoy head has to be made with a laminated construction. No glue is used here; instead, the profiles are perforated and mounted on three aluminium alloy bars running through the whole width of the float. These also provide mechanical connection with the rest of the model. The main advantage of not using glue is to keep the width of the buoy head easily changeable by adding or removing inner profiles while keeping the same two outer profiles (which are more difficult to make because of their rounded edges). For the present work, buoy head width is fixed at $300 \mathrm{~mm}$. 


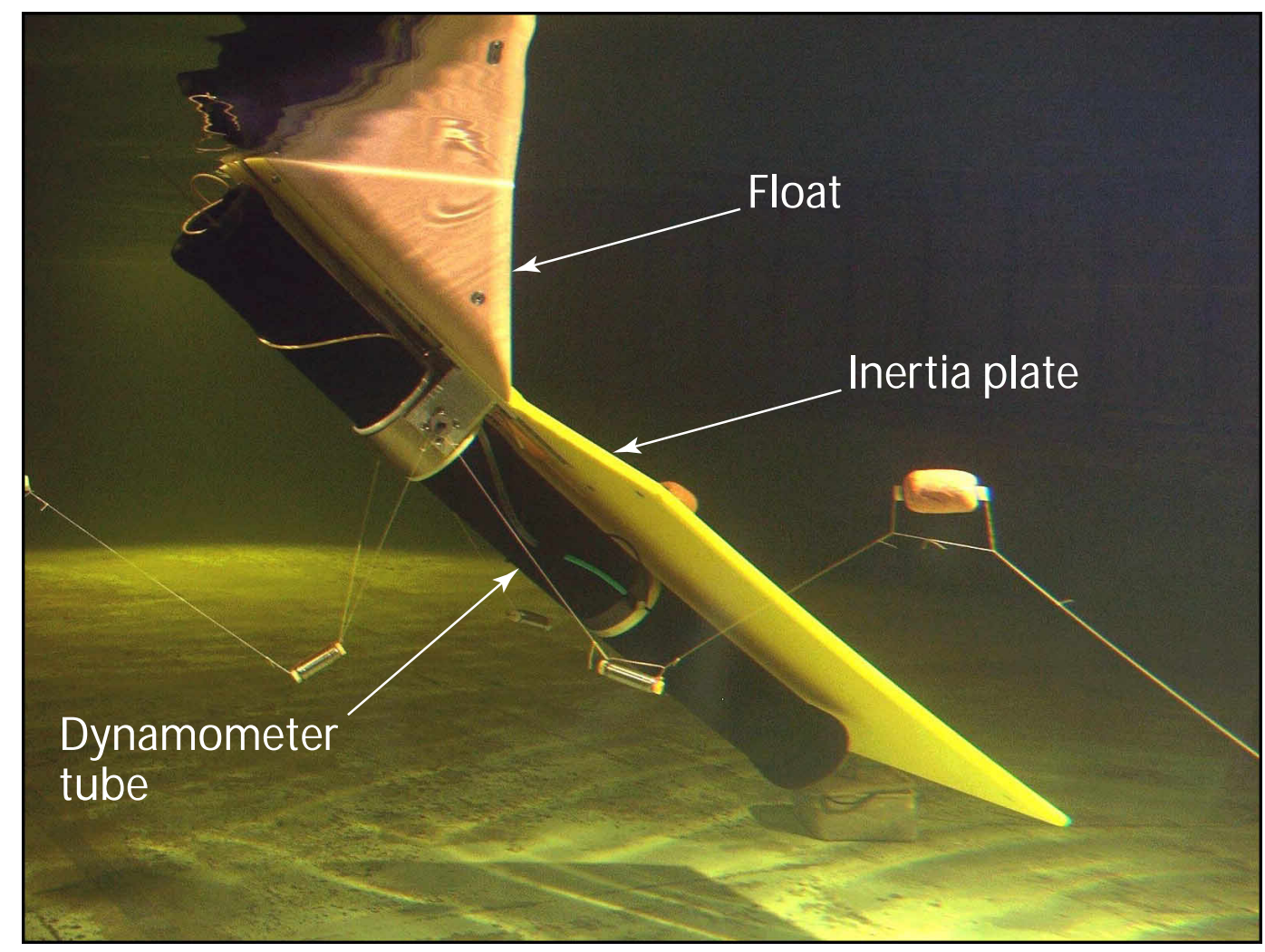

Fig. 2. Underwater view of the model in the Curved Tank. The waves come from the right.

The inertia plate is made from a $20 \mathrm{~mm}$ thick, neutrally-buoyant polyethylene plastic. It extended $900 \mathrm{~mm}$ from the end of the float and was $500 \mathrm{~mm}$ wide, but tapered in to the buoy diameter. It aims at constraining the device motion in the slope direction by providing extra added mass in the direction perpendicular to the plate.

The dynamometer simulates the power take-off mechanism of the system by relying on the 'eddy-current damper' phenomenon. Figure 3 shows a section through the dynamometer tube. The excitation coils create a magnetic field which circulates through the mild steel stator and 'return ring'. In the gap between those two parts, the field is orientated radially. The eddy-tube slides 


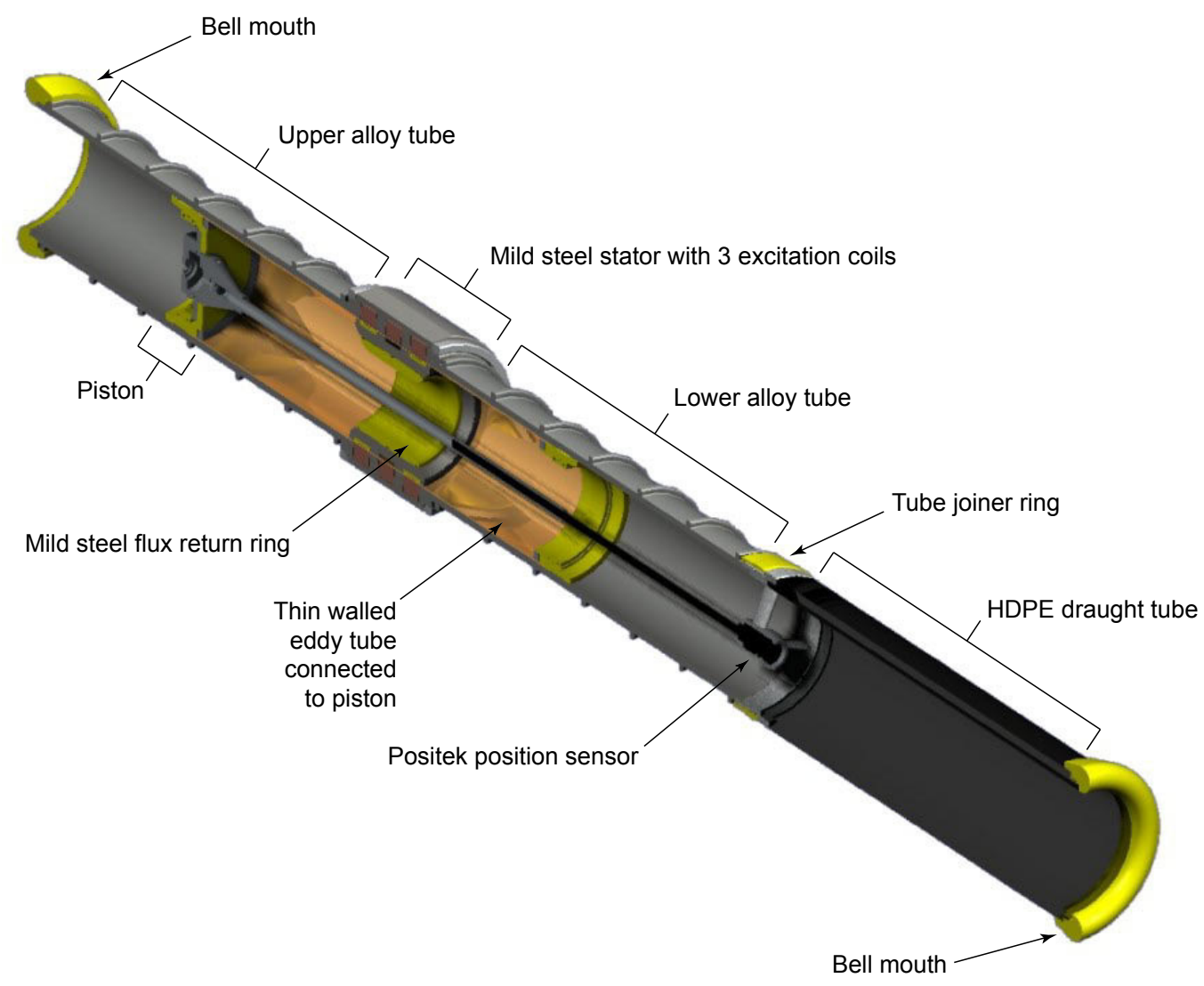

Fig. 3. Section view of the dynamometer.

axially through this $1.5 \mathrm{~mm}$ wide gap. This motion, perpendicular to the magnetic field, induces a circumferential voltage in the tube, which in turns drives a current. This current, in combination with the radial magnetic field, generates a force which tends to oppose the motion of the eddy tube. For a constant magnetic field, this force is proportional to the axial velocity of the eddy tube.

The eddy tube consists of a $1 \mathrm{~mm}$ wall thickness aluminium alloy tube. It is mated at one end to the piston.

The piston is fitted with a differential pressure sensor which provides information about the water pressure difference between the two sides of the piston, so that the net force on the piston can be known. Additionally, a special rig 
has been built to calibrate the dynamometer in order to work out the relation between the current supplied to the coils and the damping force generated.

The piston also holds one end of a tubular aluminium sheath which, as the piston moves, progressively covers and uncovers the sensor rod of the 'Positek' linear displacement transducer.

In order to locate axially the eddy tube and to enable it to slide without significant friction against the different components of the outer tube, hydrostatic water fed bearings are fitted to both ends of the eddy-tube and to the mild steel flux returning ring. The nominal radial gap between the bearings and the bored inner surfaces of the outer alloy tubes is 25 microns. More information on hydrostatic bearings can be found in (Stansfield, 1970). The significant technical effort put into these low friction bearings is motivated by the impact at model-scale of parasitic losses on power capture measurements.

In both full-scale and the present model conditions, it can be considered that the dominant restoring force in the water wave propagation phenomenon is gravity. This assumption becomes questionable in the case of very short waves (wavelength $\leq 0.1 \mathrm{~m}$ (Falnes, 2002)) where surface tension is not negligible, but this is beyond the operating range of the Curved Tank. For deep water conditions the dispersion relation yields:

$$
\lambda=\frac{g \mathrm{~T}^{2}}{2 \pi}
$$

where $\lambda$ is the wavelength, $\mathrm{g}$ the gravitational acceleration and $\mathrm{T}$ the wave period.

$\mathrm{g}$ is the same for both full scale and model conditions, so (1) is true in both 
cases. If $s$ is the geometric scale between model and full scale conditions, $\mathrm{T}$ thus scales with $\sqrt{s}$. The dimensions of power are:

$$
[\mathrm{P}]=\frac{[\mathrm{M}][\mathrm{L}]^{2}}{[\mathrm{~T}]^{3}}
$$

where $[\mathrm{M}],[\mathrm{L}]$ and $[\mathrm{T}]$ are the dimensions of mass, length and time respectively. Mass is proportional to volume, so [M] scales with $s^{3}$, and thus, power scales with $s^{3.5}$.

In the case of the sloped device investigated, the model scale is intended to be of the order of $1 / 75^{\text {th }}$, corresponding to a full-scale width of around $40 \mathrm{~m}$. For North Atlantic conditions, wave power is below 200kW per metre of wave front, $90 \%$ of the time (Mollison et al., 1976). So assuming a similar fullscale power limit, the power take-off system rating of the model should be of the order of $1 \mathrm{~W}$. This means that in order to simulate full-scale behavior accurately, power dissipation due to friction losses in the model power take-off, should be kept very low, ideally, down to the 'milliwatt' level.

\subsection{The video motion tracking device}

The movements of the model are recorded using a video motion tracking device. This method provides real time displacement information for the six degrees of freedom of the model without mechanically interfering with it.

The measurement technique relies on the principle of triangulation. Several infrared-sensitive cameras (at least two) are set to view the area where the model moves. The measurement volume is defined by the overlap of the respective camera fields of view. The cameras detect the position of small markers 
attached to the model. Two-dimensional position data from each camera are sent to a central processing unit, where they are processed, together with information about camera locations, to compute the three-dimensional co-ordinates of the markers.

The practical spacial accuracy achieved for these measurements is $0.1 \mathrm{~mm}$. The sampling frequency adopted is $32 \mathrm{~Hz}$.

\section{Comparison of numerical predictions against experimental mea- surements}

Numerical simulation of the experimental model behaviour in regular waves is done in two stages.

Comparison between numerical predictions and experimental measurements is first established with the power take-off mechanism disabled. This intermediate stage is useful to check the modelling of the device as a simple free-floating rigid body. In a second stage, the power take-off is enabled and the output of the numerical model is compared with corresponding experimental data.

\subsection{Configuration without power take-off}

The experimental model is similar to the one described in section 2.2 with the difference that the dynamometer is not fitted with a piston and therefore forms a simple hollow tube. Two configurations are explored:

- The dynamometer tube is open at both ends, thus allowing water to flow in and out of the tube. 


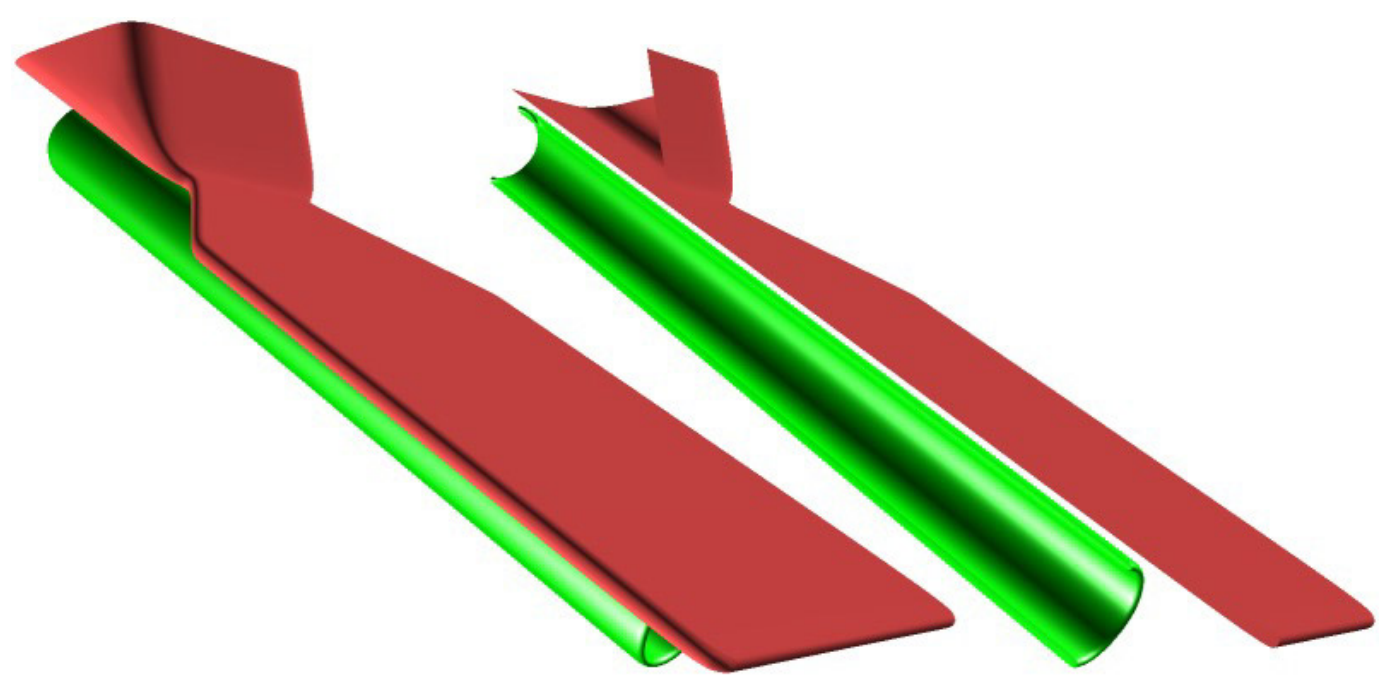

Fig. 4. Rendered view (left) of the geometry corresponding to the configuration with the tube open at both ends. The section view (right) show the representation of the tube.

- The tube is filled with water and blocked at both ends.

These two configurations can be seen to approximate, respectively, to notional 'zero damping' and 'infinite damping' settings on the model power take-off.

The geometrical representation of the experimental model that is input to WAMIT was designed using the CAD package MultiSurf. Figure 4 shows solid views associated with the representations of open and blocked tube configurations.

The comparisons between numerical predictions and experimental data are given for the motion of a point fixed with respect to the device. This point is located on the plane of symmetry of the system and slightly above the float. The location of this point and the configuration of the device are shown in figure 5 . 


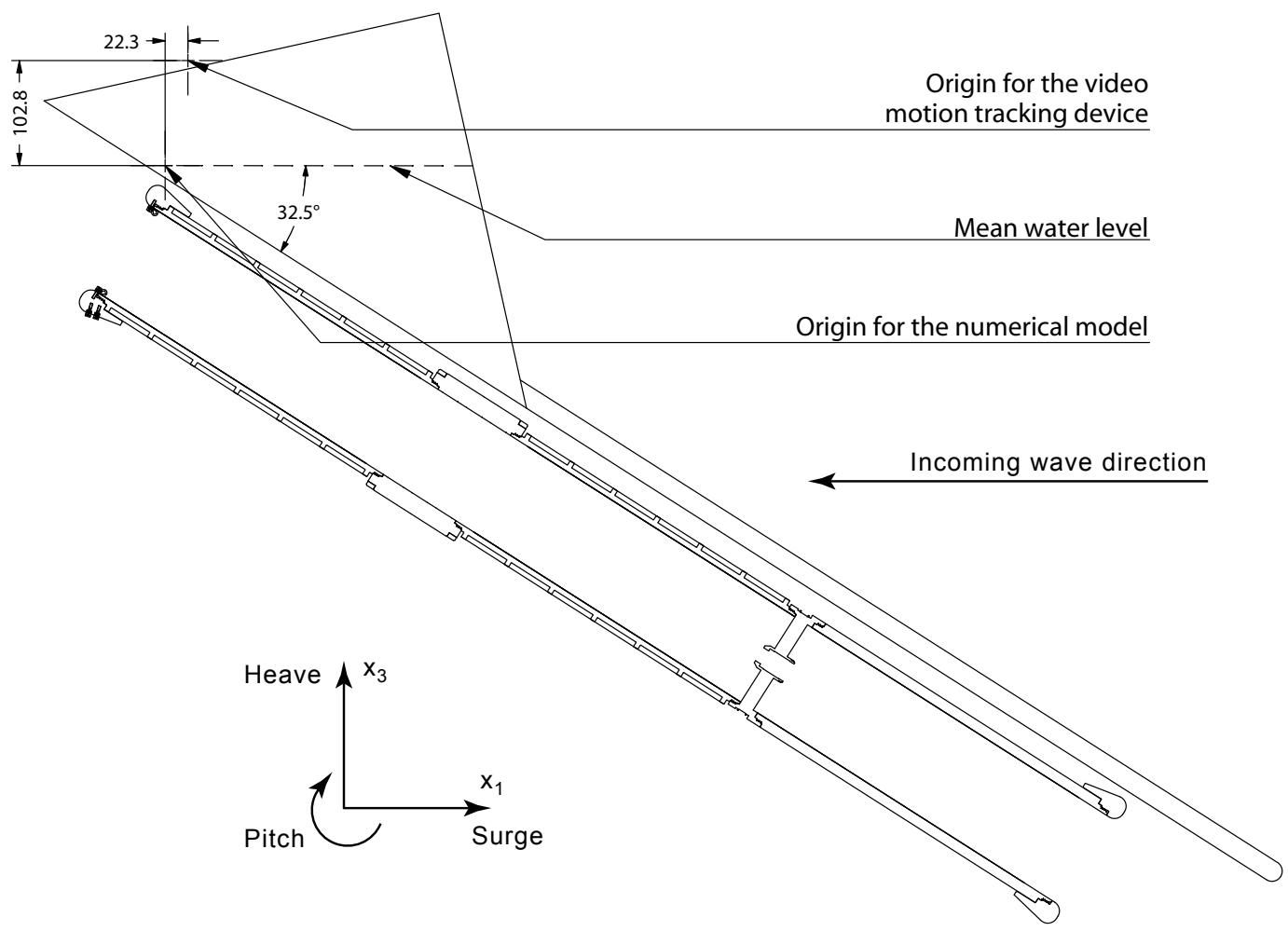

Fig. 5. Section view of the device with the tube shown open. The point used for motion comparison is the origin for the video tracking system. Distances are indicated in $\mathrm{mm}$.

\subsubsection{Roll instability}

Given that the device is excited by waves propagating in a direction parallel to its plan of symmetry, it should only respond in surge, heave and pitch. However, experimental observations indicate that the device responds significantly in roll, particularly at periods around $1.2 \mathrm{~s}$, as shown in figure 6 .

For the open tube configuration, the roll response is close to zero except for periods in the vicinity of $1.2 \mathrm{~s}$ and $2 \mathrm{~s}$. In the former case there is a sharp peak, in the latter, response values are much lower and the peak more spread out. The blocked tube configuration exhibits similar behaviour but with both peaks slightly shifted towards longer periods. 

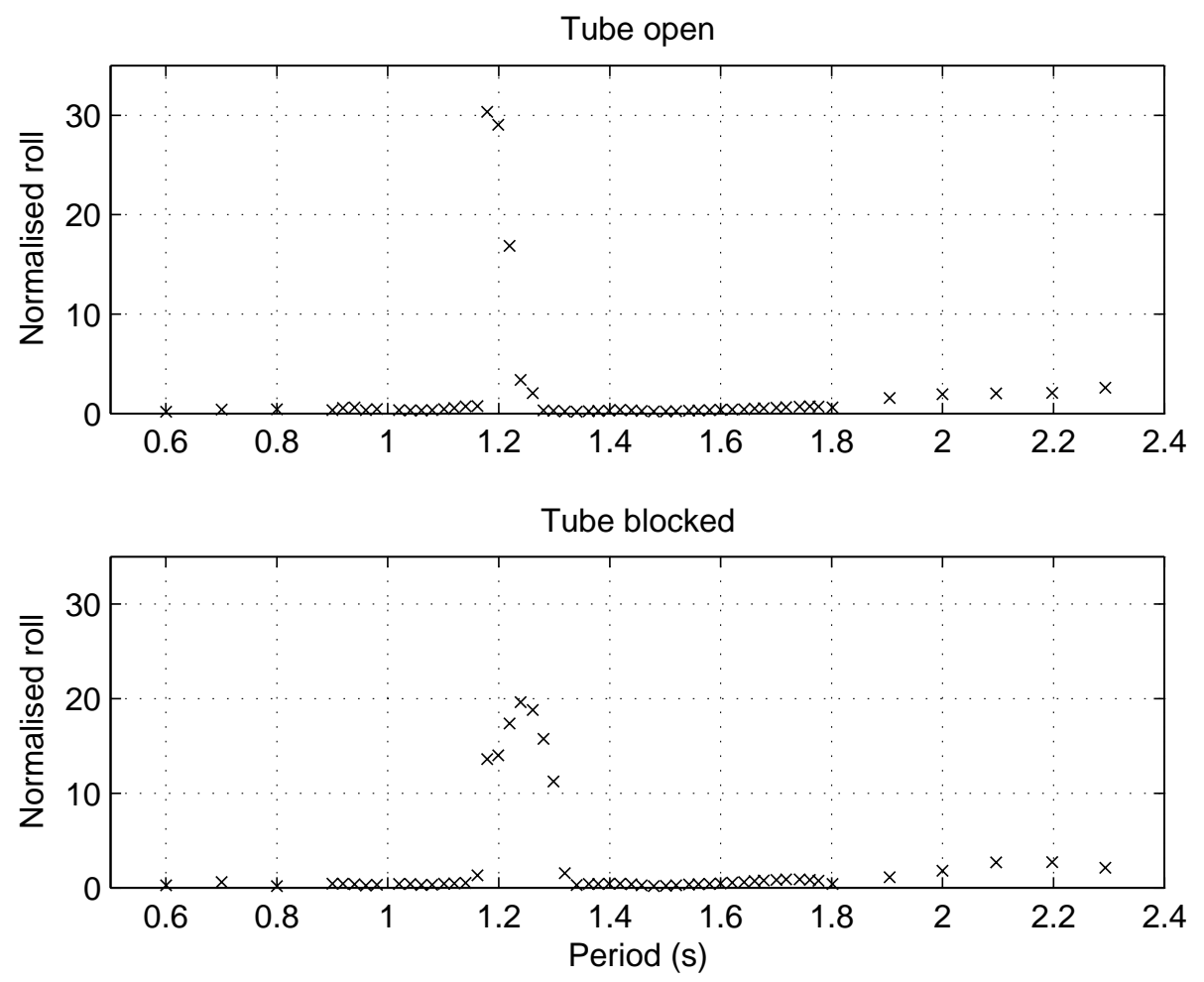

Fig. 6. Response in roll (in degrees) normalised by wave amplitude plotted against wave period for both experimental configurations. The amplitude is computed using root mean square.

Further analysis reveals that the frequency of the roll oscillations for the first peak does not correspond to the frequency of the waves which the device is subjected to. Figure 7 shows the frequency distribution of the roll response obtained by Fast Fourier Transform (FFT) for the open tube configuration in waves of $0.848 \mathrm{~Hz}(1.18 \mathrm{~s})$ and the blocked tube configuration in waves of $0.781 \mathrm{~Hz}(1.28 \mathrm{~s})$. These frequencies correspond to the sharp peaks shown on figures 6 . It should be noted that here, the horizontal axis is in frequency rather than period as this is more appropriate for an FFT.

From figure 7, it appears that the dominant frequency of the roll response is 

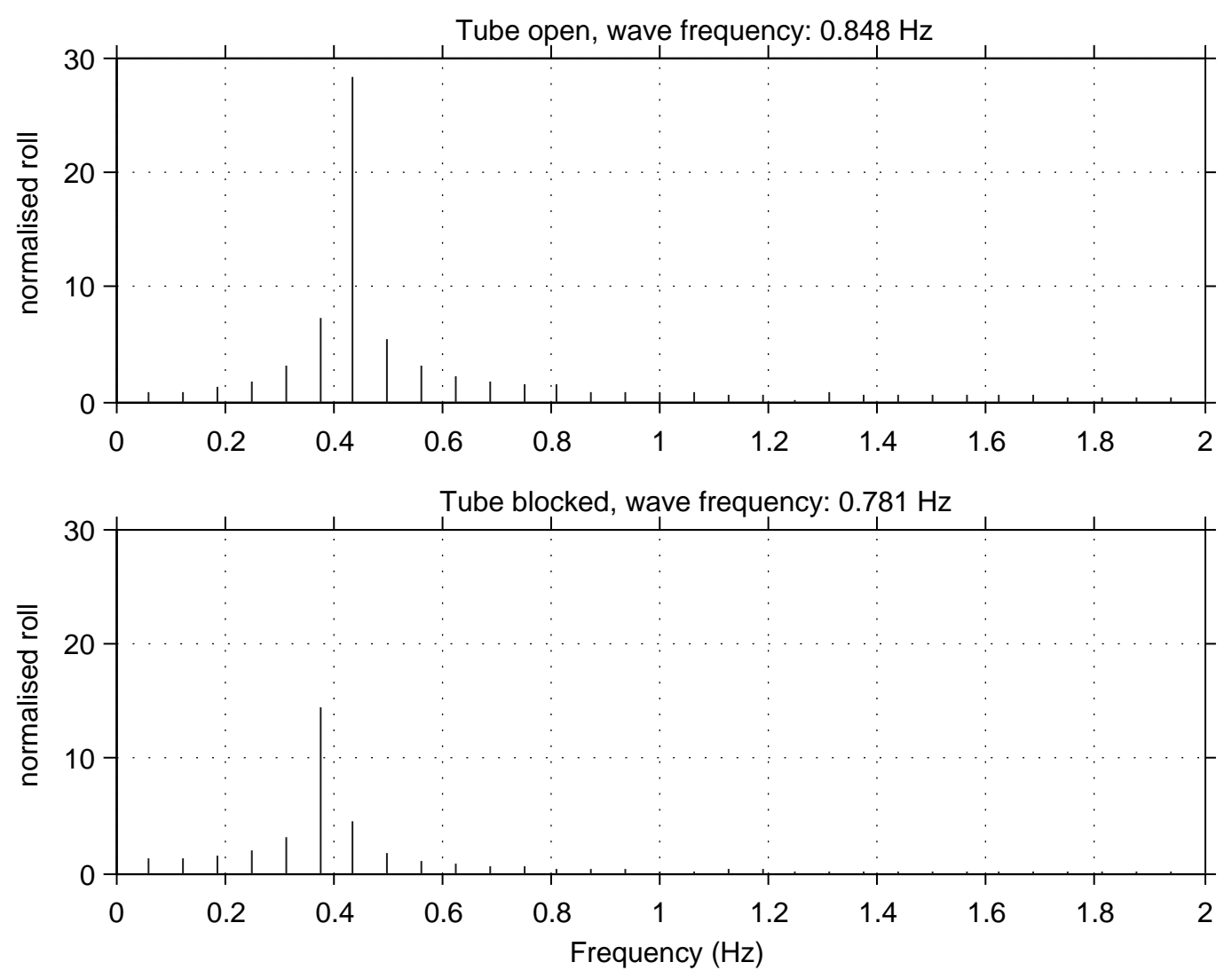

Fig. 7. Frequency distribution of the roll response for the open (top) and blocked (bottom) tube configurations in waves of $0.848 \mathrm{~Hz}$ (top) and $0.781 \mathrm{~Hz}$ (bottom).

about half of the exciting wave frequency. This clearly non-linear phenomenon is believed to be related to indirect roll excitation by heave and pitch motion. This type of behaviour was originally reported by Froude in 1863 in the case of ships (Froude, 1955).

For the sake of simplicity, consider the interaction only between heave and roll. As mentioned earlier, for reason of symmetry, there is no direct roll excitation from the wave. If the body is subjected to a small perturbation in roll however, the roll hydrostatic restoring moment is affected by heave and free surface motions. Liaw (1994) has shown that when considering small roll angles, the hydrostatic roll restoring moment can be considered as the sum of three terms: 
- a constant term that can be derived from the body position at rest

- a first order term in relative heave motion

- a second order term in relative heave motion.

The relative heave motion is defined as the absolute heave motion minus the water surface elevation.

This heave-roll coupling through the roll hydrostatic restoring moment induces instability in the roll equation of motion for certain values of heave amplitude and incoming wave period. Depending on the hydrostatic and hydrodynamic characteristic of the body, the range of wave period and heave amplitude over which the instability region spreads can vary. The case where the exciting wave period is about half the roll resonance is however known to be critical in terms of instability (Liaw, 1994).

In the case of the experimental model, it is likely that small cross waves caused by reflections on the tank boundaries have hit the model sideways. This would then induce the initial roll perturbation that triggers the roll instability.

For the open and blocked tube configurations, the roll resonance period has not been measured experimentally. Numerical computations were, however, carried out in the case where the direction of propagation of the waves is perpendicular to the model plan of symmetry. The response in roll is shown on figure 8 .

For both configurations, the values reached by the resonance peaks are nonphysical. This can be attributed to the neglect of viscous damping in the computation. These results indicate resonance periods that are close to the experimental roll oscillation periods reported in figure 7 . This tends to confirm 

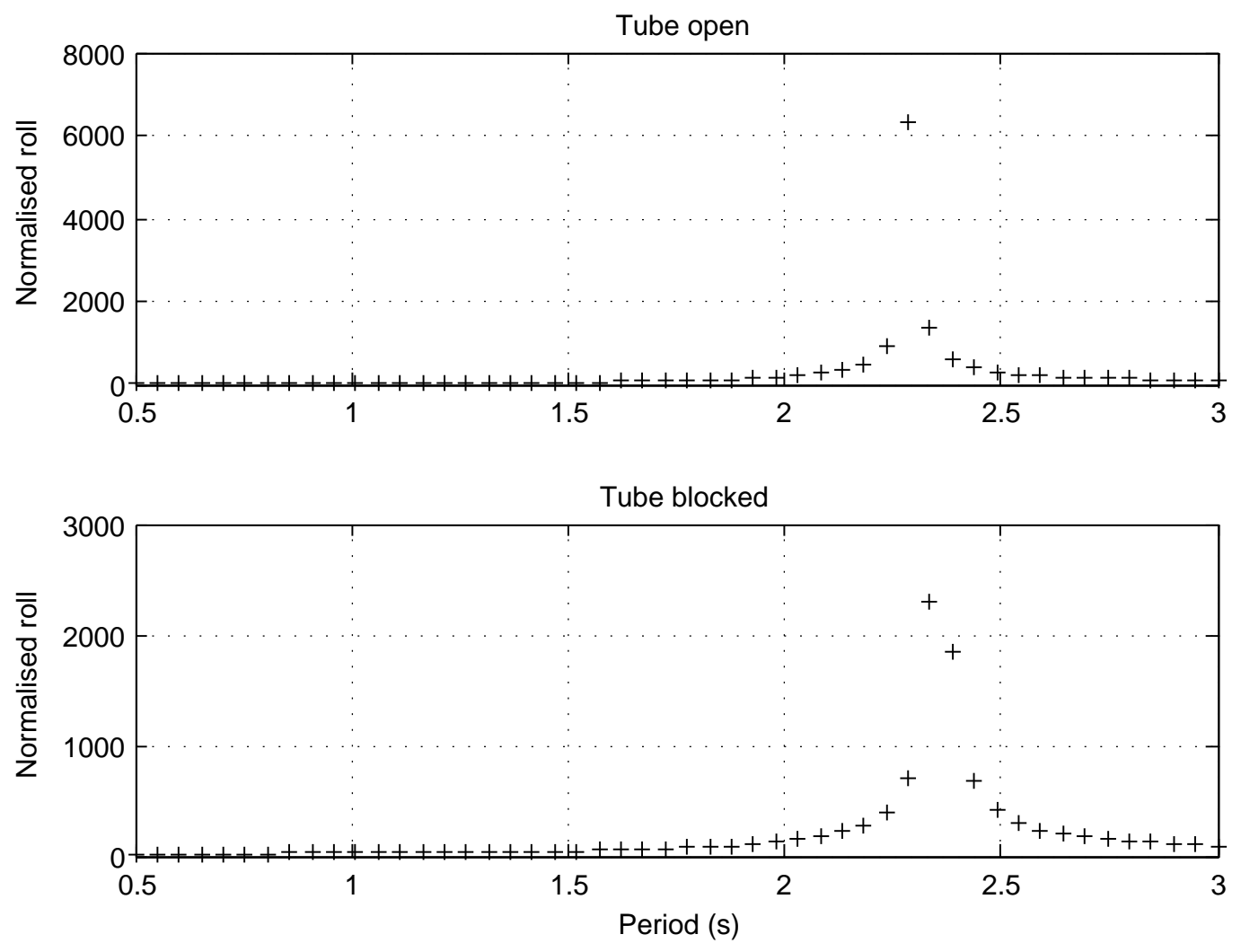

Fig. 8. Numerical predictions of roll response (in degree) normalised by wave amplitude with waves travelling perpendicularly to the plane of symmetry of the body. that the roll motions shown in figure 6 are caused by non-linear coupling of roll with heave and pitch via roll stiffness.

In WAMIT, the roll restoring moment is assumed constant and computed from the rest position of the body. It thus cannot predict roll response associated with this roll instability.

\subsubsection{Plane motion}

The experimental data used for comparison with numerical predictions correspond to motion in the $\left(x_{1}, x_{3}\right)$ plane (see figure 5 ). For each wave period investigated, motions are recorded after allowing one minute for the system 
to reach steady state.

The comparison in terms of amplitude is shown on figure 9.

In terms of heave, the agreement is generally good for both configurations. The amplitude of the resonance peaks predicted by WAMIT is however much higher than what is observed experimentally. This type of discrepancies can be attributed to the neglect of viscous damping by the numerical model.

It can also be noticed that the resonance peak occurs at a longer period for the blocked tube configuration than for the open tube. The blocked tube is filled with water, the mass of the model in this configuration is therefore higher than with the open tube. Since both configurations have the same water plane area, this extra inertia shifts the resonance towards longer periods.

In long waves, both experimental and numerical results converge, as expected towards 1 .

In terms of surge, numerical predictions and experimental data exhibit the same trend. It can be noted however that the experimental points seem to 'fluctuate' around the numerical curve with an oscillating pattern. This 'ripple noise' phenomenon is believed to be primarily caused by reflections from the tank boundaries. Depending on the wavelength, the reflected wave acts constructively or destructively with the incident wave at the point of the model. This interpretation is suggested by the fact that the cyclic fluctuations of the experimental points about the local mean are more rapid for shorter waves than for longer waves. For short waves, a relatively small change in frequency causes a relatively big change in the phase of the reflected wave at the model position. 

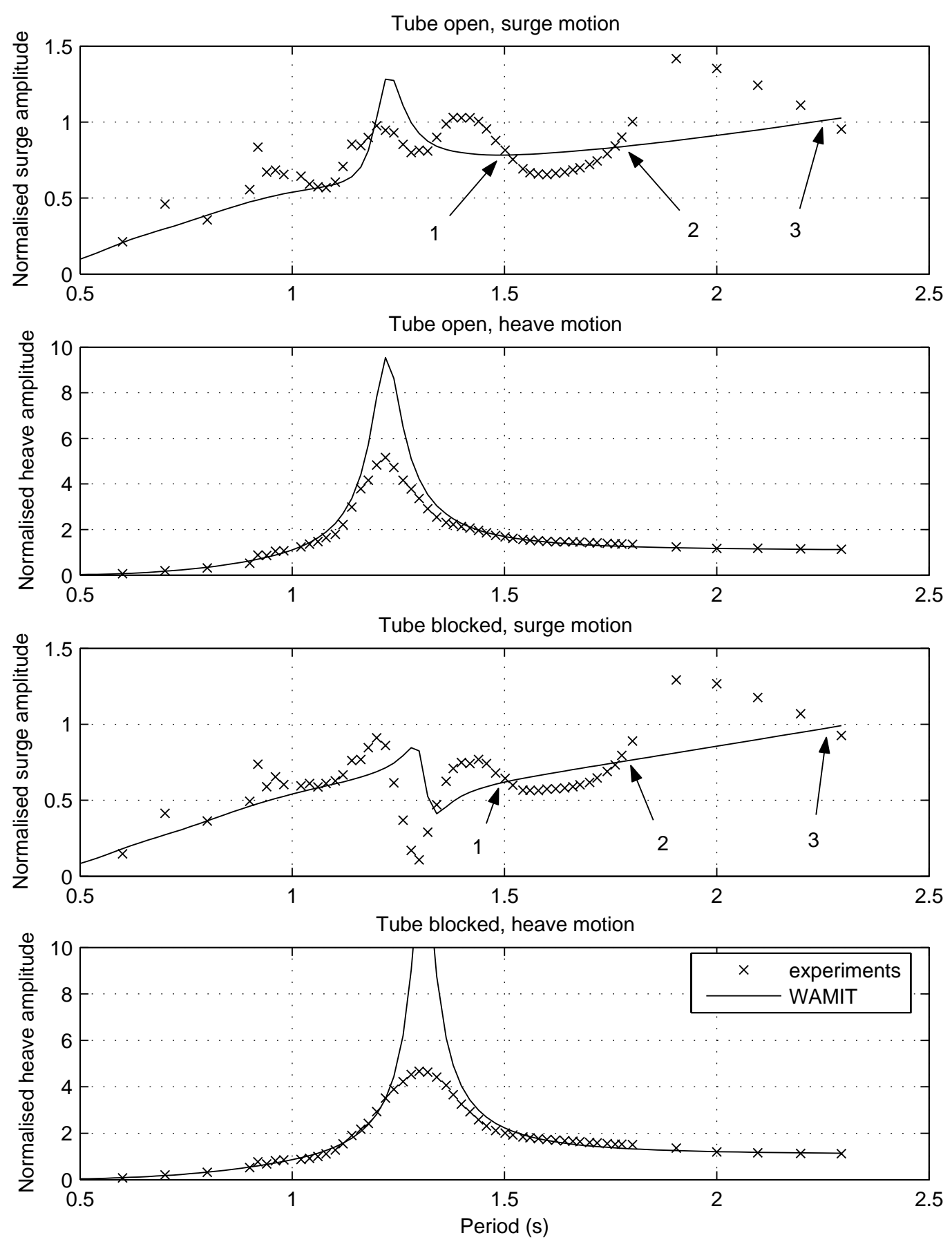

Fig. 9. Comparison between numerical predictions and experimental data for surge and heave motions of the open and blocked tube configurations. The motion amplitudes are normalised by wave amplitudes. Please refer to text for explanation of significance of numbered points. 
This interpretation can be quantitatively verified for both configurations by considering the fluctuations of the experimental points with respect to the numerical curve between wave periods $1.5 \mathrm{~s}$ and $2.3 \mathrm{~s}$. (that is between point 1 and 3 on the surge graphes of figure 9). Let $d$ be the total distance from the model to the reflecting surface and then back again to the model. $d$ thus corresponds to twice the distance from the model to the back of the tank. $d$ can be expressed from the wavelength by introducing a coefficient $a$ so that:

$$
d=a \lambda
$$

Between points 1 and 3 (in figure 9), an entire pseudo-oscillation of the experimental data is taking place. It would thus be expected that for the wave regime of point 1 there is one more wavelength within $d$ compared with the wave regime of point 3 . In other words, if

$$
d=a \lambda_{3}
$$

then

$$
d=(a+1) \lambda_{1}
$$

where $\lambda_{1}$ and $\lambda_{3}$ are the wavelengths for wave regimes 1 and 3 respectively. Similarly, for wave regime 2 , there should be half a wavelength more within $d$ than for wave regime 3 . That is, if

$$
d=a \lambda_{3}
$$

then

$$
d=(a+0.5) \lambda_{2}
$$


The validity of this interpretation can be checked by comparing the value of $d$ obtained from equations (4) and (5) and the one obtained from equations (6) and (7). For that purpose, $\lambda_{1}, \lambda_{2}$ and $\lambda_{3}$ are calculated from wave periods and water depth using the dispersion relation of linear wave theory (see equation (24), p.244 of (Newman, 1977)):

$$
\begin{aligned}
\lambda_{1} & =3.43 m \\
\lambda_{2} & =4.46 m \\
\lambda_{3} & =6.69 m
\end{aligned}
$$

(4) and (5) yield

$$
d=7.04 m
$$

and (6) and (7) yield

$$
d=6.69 m
$$

The small discrepancy between (11) and (12) can be explained by the fact that the mean drift force is probably different for wave regimes 1,2 and 3 . Given the compliance of the mooring this should result in slightly different average position of the model when the steady state is reached. These values of $d$ are also consistent with the position of the model in the tank. As shown on figure 1 , the model was located approximatively $3.5 \mathrm{~m}$ away from the rear wall, which corresponds to a nominal value of $d$ equal to $7 \mathrm{~m}$.

It can be noticed that the ripple noise phenomenon does not affect the heave measurements. The motion amplitudes shown in figure 9 are normalised by the measured wave amplitude. This makes the heave results be fairly independent of wave reflection. However this is not the case for surge. 
To understand this, it is useful to consider the simplified case where the floating model is small with respect to the wavelength and located in an unbounded wave field. In this situation, the heave response will follow the motion of the free surface, so when the model is subjected to a regular incident wave and no reflected wave, its normalised heave response will be 1 . Similarly, from the small body approximation the surge response will follow the horizontal motion of the water particles. As these have a circular motion in deep water waves, the normalised surge response in these conditions will also be 1 . Now consider the extreme case of a fully reflective wall behind the model. This configuration will generate a standing wave pattern. The heave response of the model will depend on its location on this pattern. For instance, if it is located at an antinode, the heave motion will be twice what it would be in the absence of reflected wave. However, the normalising process will yield a normalised heave response of 1 , since at the antinode, the wave amplitude is twice what it would be without reflection. This argument holds true for any location of the model, so the normalised heave response of this small model is not affected by its location on the standing wave pattern.

On the other hand, the location of the model will affect the surge response. For example, if located at an antinode, the model will not have any surge motion. If located at a node, the model surge response will be twice what it would be without reflection. The normalising process exaggerates this discrepancy further. In the vicinity of an antinode, the next-to-zero surge motion amplitude is divided by twice the regular wave amplitude. Near a node, the doubled surge amplitude is divided by a wave amplitude close to zero. Shallow water waves increase the discrepancy. In these conditions, the surge motion in regular wave is larger although it remains zero at an antinode of the standing wave. 
In the case of the blocked tube, the prominent feature of the surge response predicted by WAMIT is the step change occurring at a period of about 1.3s. This feature seems to also exist in the experimental data but at a slightly shorter period. Given the level of noise associated with the experimental measurements, it is difficult to tell whether this step change is only an artefact caused by the noise or if the discrepancy is real and reveals a more fundamental problem.

The above motion comparison provides useful information on the correlation between numerical predictions and experimental data in terms of amplitude. In order to establish a comparison in terms of phase, the phase shift between surge and heave responses is compared. The results are presented qualitatively by plotting the averaged experimental ellipsoids described by the point specified in figure 5 and the corresponding WAMIT computations for a set of wave periods. Figure 10 shows the comparison for the open tube configuration and figure 11 for the blocked tube one. It should be noted that scale of the axes varies through the period range. This is because the amplitude of the ellipsoids varies greatly through the period range. Moreover, these figures are aimed at providing qualitative information. Quantitative amplitude comparisons can be obtained from figure 9 .

For both configurations, the agreement is generally good. In terms of phase shift, this good correlation is indicated by the alignment of the main axis of both the experimental and numerical ellipsoids. A few discrepancies are however visible:

- For $0.6 \mathrm{~s}$ wave period, the experimental averaged ellipsoids are significantly distorted, especially in the case of the open tube. As explained earlier this is a consequence of the drift in surge. Nevertheless, phase shifts match, as 

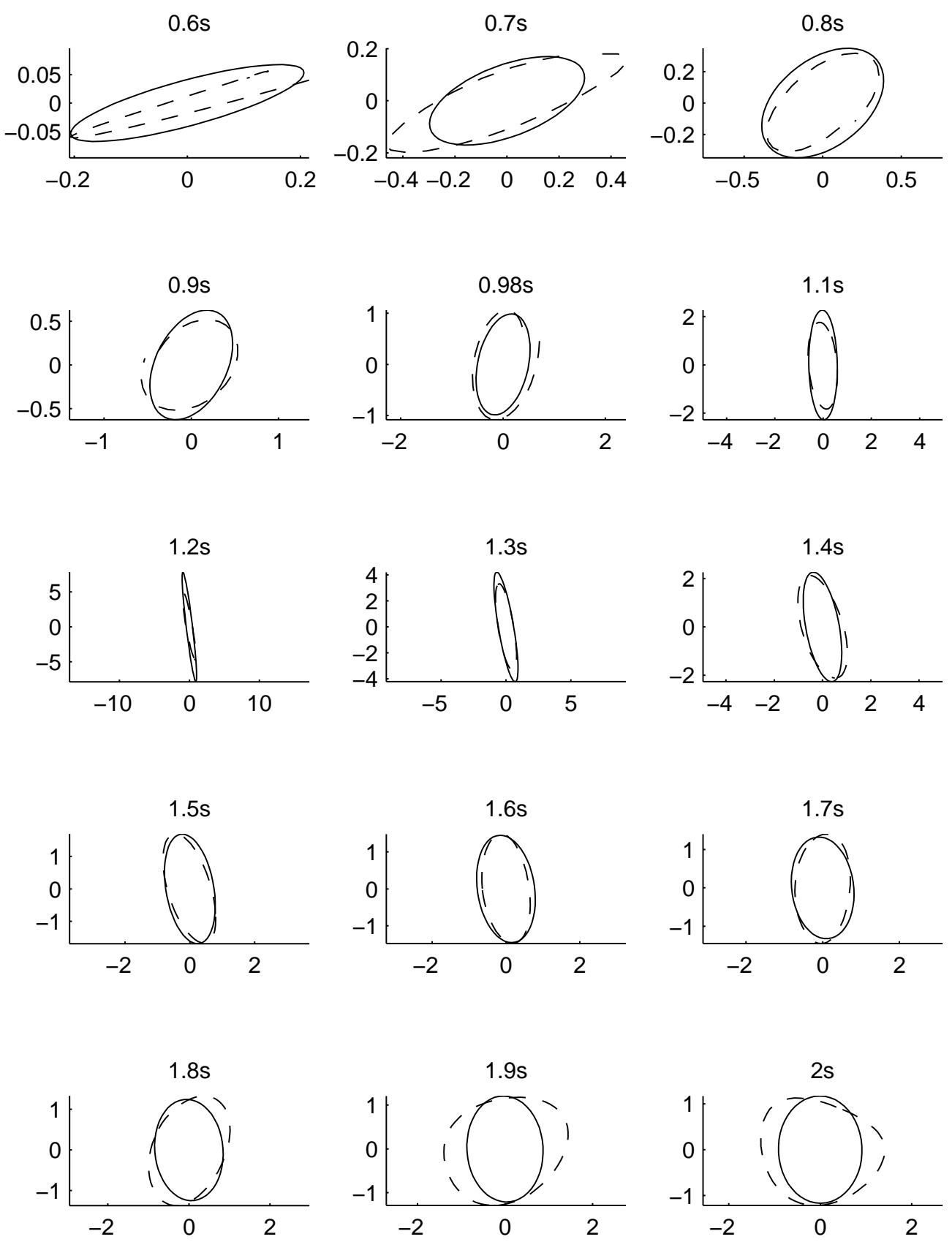

Fig. 10. Trace of the motion in the $\left(x_{1}, x_{3}\right)$ plane for the open tube configuration with wave maker gain set to 2 . The dashed lines correspond to the experimental measurements and the solid lines to the numerical predictions. The scale of both axes of the graphs are the same to preserve the aspect ratio. The displacements shown are normalised by wave amplitude. Wave period is indicated above each graph. When looking at the plots, waves are coming from the right. 

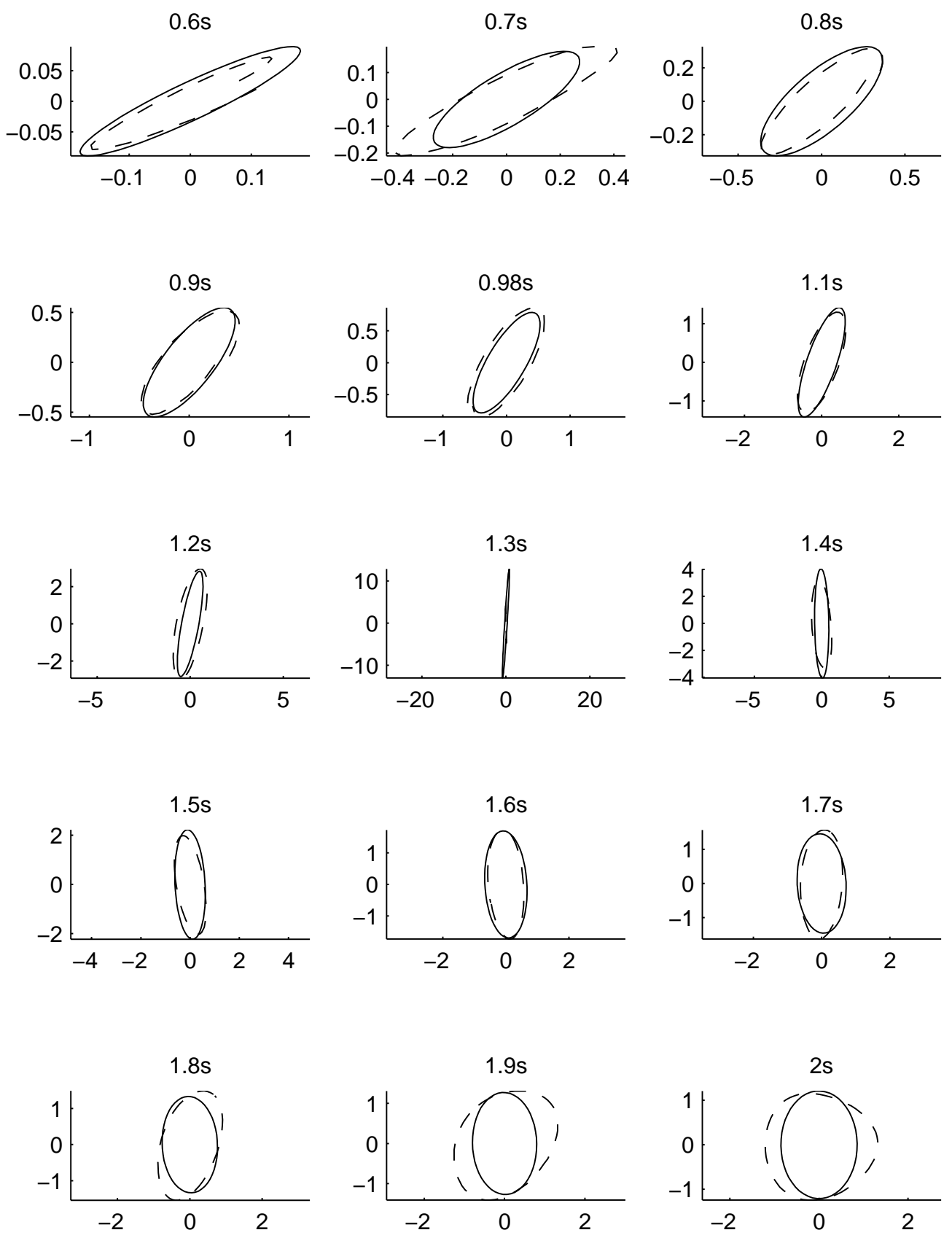

Fig. 11. Trace of the motion in the $\left(x_{1}, x_{3}\right)$ plane for the blocked tube configuration with wave maker gain set to 2 . The dashed lines correspond to the experimental measurements and the solid lines to the numerical predictions. The scale of both axes of the graphs are the same to preserve the aspect ratio. The displacements shown are normalised by wave amplitude. Wave period is indicated above each graph. When looking at the plots, waves are coming from the right. 
shown by the alignment of both axes.

- For 0.7s wave period, the experimental ellipsoids are more elongated than their numerical counterparts. When looking at the corresponding point (second from the left) in figure 9, this is associated with a surge response about $50 \%$ higher than the numerical prediction. This discrepancy is consistent with the ripple noise mentioned earlier. The phase shift correlation is however good.

- For the three last wave periods - 1.8, 1.9 and $2 \mathrm{~s}$ - the phase shift agreement deteriorates as waves become longer and the shape of the experimental trace drifts away from that of an ellipse. This is not due to the surge drift mentioned earlier and indicates a non-linear contribution to the motion.

\subsection{Configuration with power take-off}

The experimental model is the one described in section 2.2. The numerical model integrates the power take-off formulation derived in the companion paper.

As for the configuration without power take-off, MultiSurf is used to generate a geometry. Figure 12 shows solid view representations of the input geometry.

As for the configuration without power take-off, comparisons between numerical predictions and experimental data are given for the motion of a point fixed with respect to the device.

The relative motion of the piston with respect to the surrounding tube is also compared. 


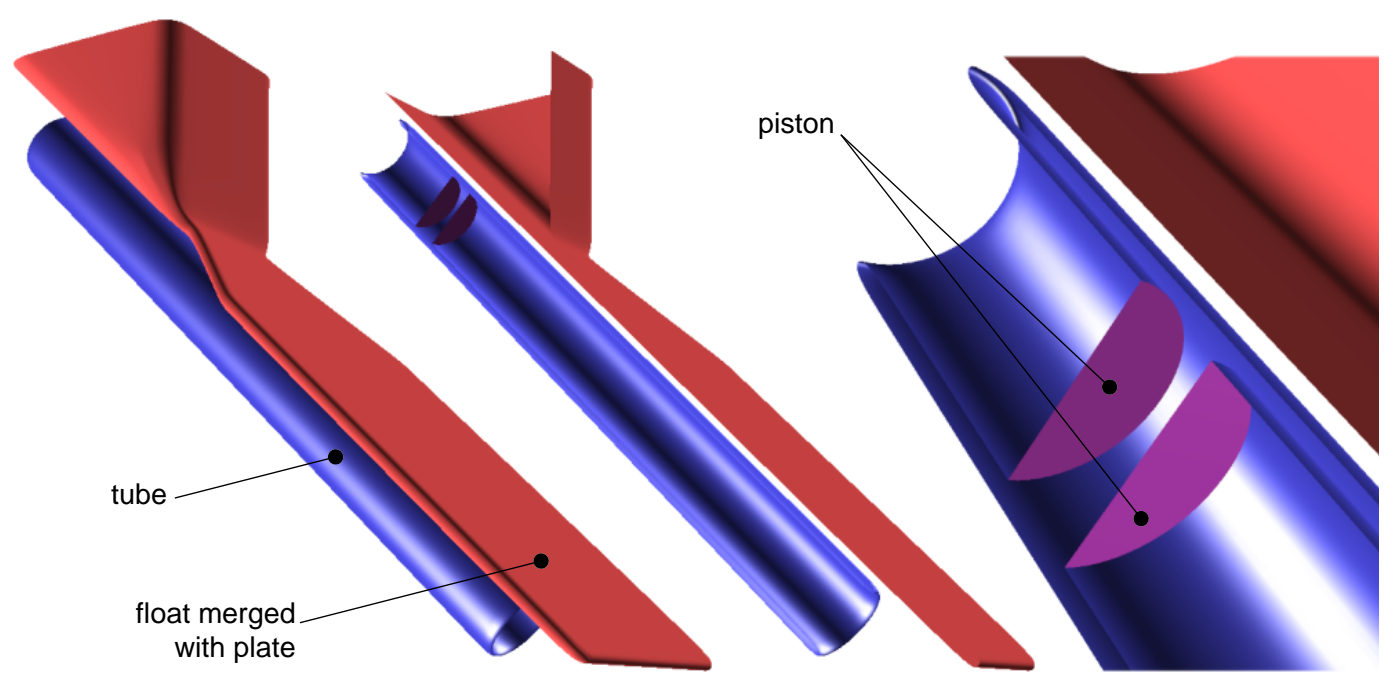

Fig. 12. Solid views of the input geometry for configuration with power take-off. The section view (centre) and the close up (right) show in detail the representation of the piston within the tube.

Experimental measurements were carried out over a range of wave periods and wave amplitudes for different values of damping applied to the piston motion.

\subsubsection{Roll instability}

The configuration with power take-off exhibits a similar roll instability to that of the free-floating configuration without power take-off. This would be expected given the similarity of the geometries of both configurations.

\subsubsection{Plane motion}

The comparisons are shown in figure 13 for four different values of dynamometer damping: $3.45,14.17,27.52$ and $40.7 \mathrm{~kg} . \mathrm{s}^{-1}$.

In the surge results, for the four damping values, numerical predictions and experimental data exhibit similar trends. As in section 3.1.2 however, the 
Surge
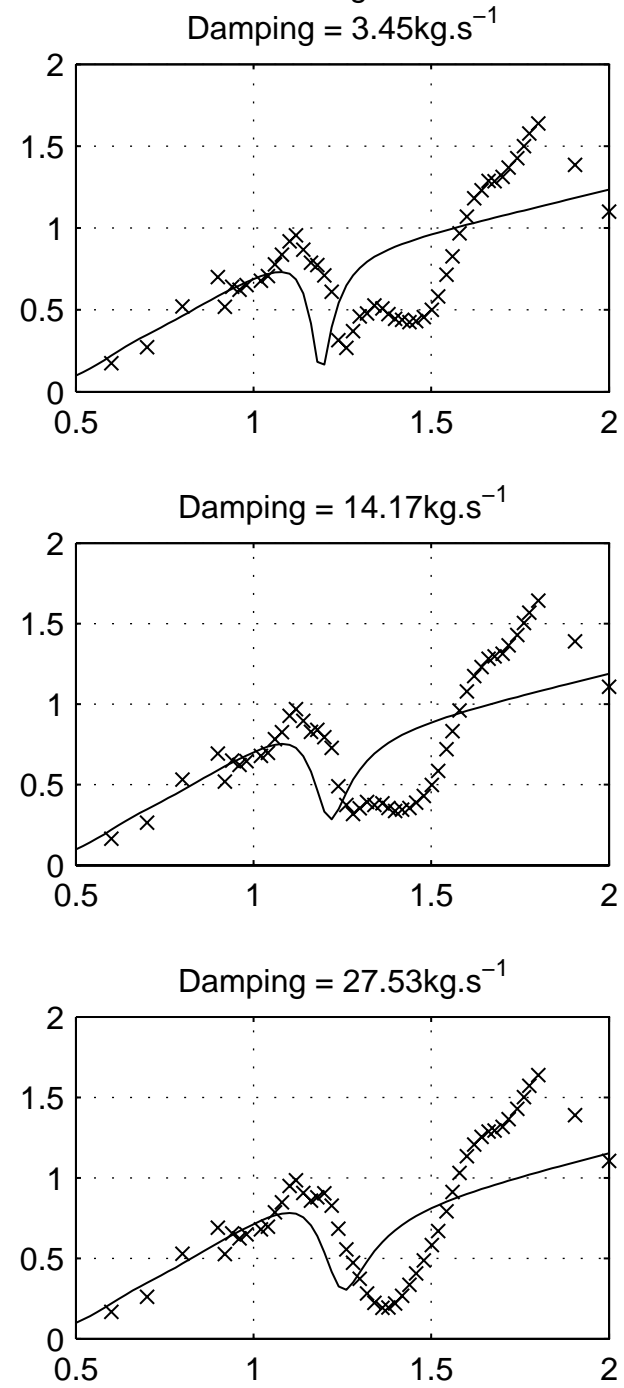

Damping $=40.7 \mathrm{~kg} \cdot \mathrm{s}^{-1}$

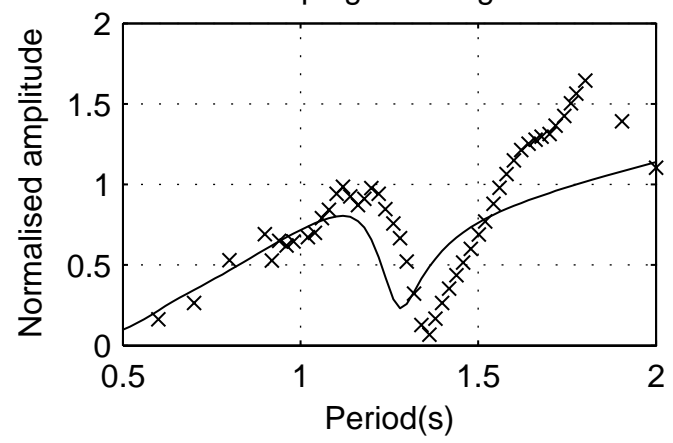

Heave

Damping $=3.45 \mathrm{~kg} \cdot \mathrm{s}^{-1}$

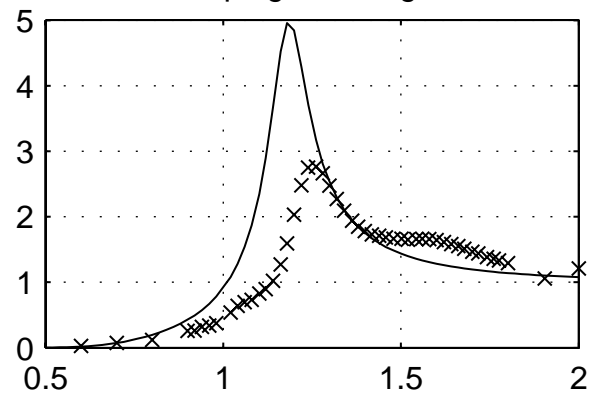

Damping $=14.17 \mathrm{~kg} \cdot \mathrm{s}^{-1}$

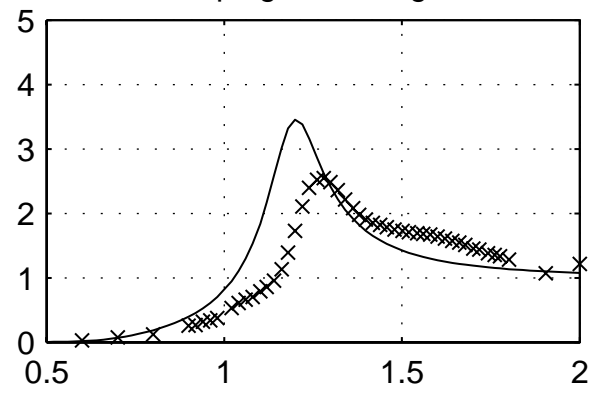

Damping $=27.53 \mathrm{~kg} . \mathrm{s}^{-1}$

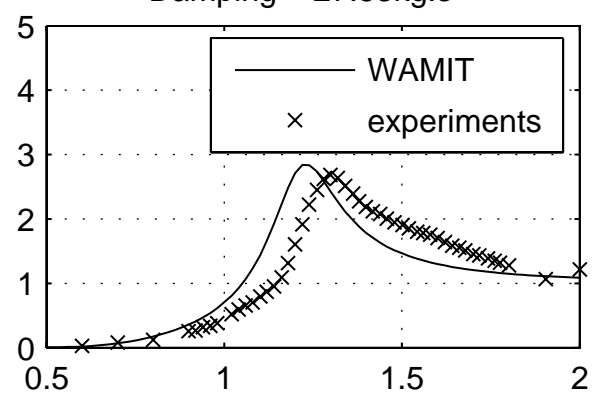

Damping $=40.7 \mathrm{~kg} \cdot \mathrm{s}^{-1}$

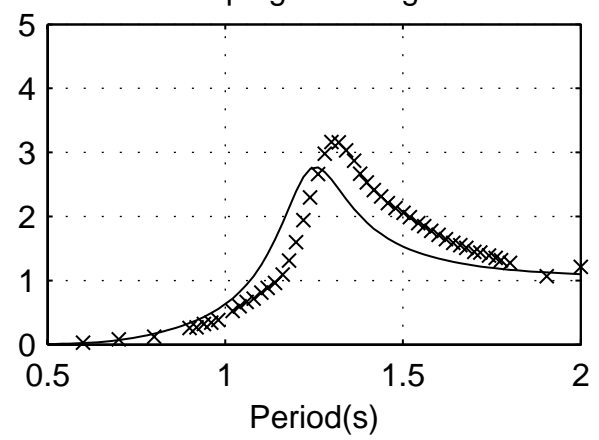

Fig. 13. Comparison between numerical predictions and experimental data for surge and heave motions for four values of the dynamometer damping. For each case, the damping value applied to the piston motion is indicated above the graph. 
experimental measurements are contaminated with ripple noise. Also, both experimental points and numerical curves plunge sharply for wave periods in the vicinity of $1.2 \mathrm{~s}$. There is however a small relative offset of about $0.06 \mathrm{~s}$ in the wave period for which this plunge takes place.

In the heave results and for the four damping values, the general trend of both experimental data and numerical curves is similar in that they both display a peak. This peak occurs at the same wave period as the plunge in surge. As in surge, there is a discrepancy of about $0.06 \mathrm{~s}$ in the wave period for which this peak occurs. The amplitude of the peak of the numerical prediction is about $65 \%$ higher than the experimental one for the lowest damping case. As

the damping increases, this discrepancy in amplitude reduces and for damping values of 27.53 and $40.7 \mathrm{~kg} . \mathrm{s}^{-1}$, peaks amplitudes of experimental and numerical data are very similar. A possible interpretation for this phenomenon is that WAMIT does not take into account viscous damping. This can lead to predictions of very large responses at resonance. This is probably the case for the lowest damping setting. As the dynamometer damping is increased however, the viscous damping could become less significant with respect to the dynamometer damping. In other words, the overall damping in the physical experiment would be largely dominated by the dynamometer damping. This would yield a better amplitude match with numerical predictions since the dynamometer damping is taken into account in the numerical model.

\subsubsection{Piston motion}

The comparisons are shown on figure 14 for the same four different values of the dynamometer damping. 

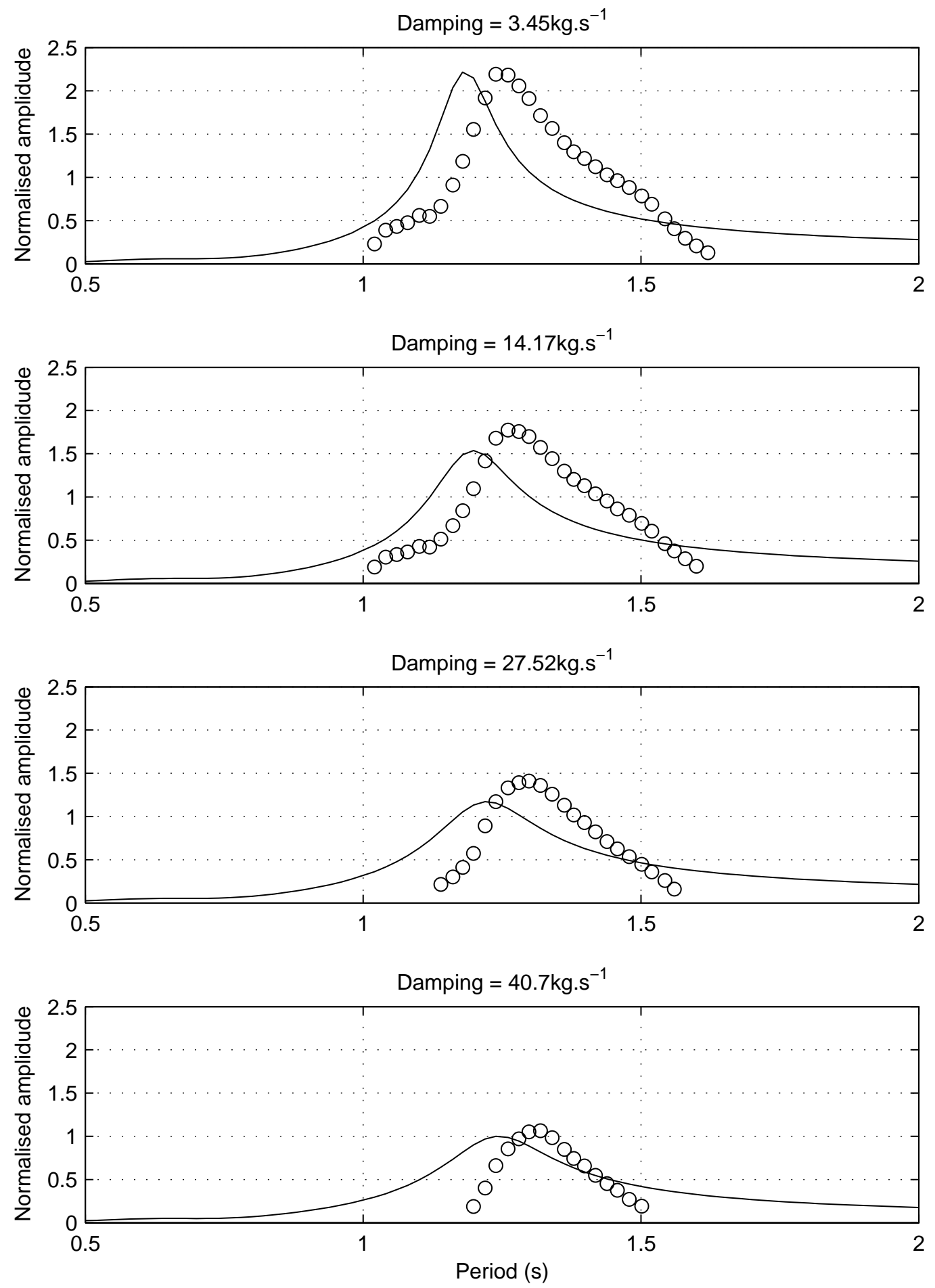

Fig. 14. Comparison between numerical predictions (solid lines) and experimental data (circles) for the piston motion relative to the tube. For each case, the damping value applied to the piston motion is indicated above the graph. 
The general trend of both experimental data and the numerical data is similar, in that they both exhibit a peak. The wave period of this peak corresponds to the peak in heave response and to the sharp plunge in surge. As for the surge and heave responses, there is a shift of about $0.06 \mathrm{~s}$ between numerical and experimental for the period at which the peak occurs.

Concerning the amplitudes of the peaks, the discrepancies do not exceed $20 \%$ and are actually much lower in the case for the $3.45 \mathrm{~kg} . \mathrm{s}^{-1}$ and $40.7 \mathrm{~kg} . \mathrm{s}^{-1}$ damping values. The evolution of these discrepancies with the dynamometer damping is however different from that of the heave response shown on figure 13.

\subsubsection{Capture width}

The capture width corresponds to the ratio of the power captured by the device to the power contained in the incoming wave front of the same width as the device. In the present case, this corresponds to the frontal width of the model float, that is $300 \mathrm{~mm}$.

The instantaneous power absorbed by the power take-off mechanism $P(t)$ is given by:

$$
P(t)=D_{\text {pto }} v_{\mathrm{p}}(t)^{2}
$$

where $D_{\text {pto }}$ is the power take-off damping and $v_{\mathrm{p}}(t)$ is the piston instantaneous velocity (relative to the tube).

The time-average power absorbed $\bar{P}$ is computed from integration of (13).

The power contained in the incoming wave fronts was derived from the mea- 
sured wave amplitude.

Comparisons between numerical predictions and experimental measurements of the capture width are shown in figure 15 for different values of the power take-off damping.

The correlation between numerical predictions and experimental measurements for the capture width is very similar to that of the piston motion (see figure 13. There is a similar discrepancy between numerical and experimental data in the period at which resonance takes place. This discrepancy is however slightly larger for the capture width than for the piston motion.

In terms of wave energy conversion performance, these results are rather disappointing. The capture width does not exceed $45 \%$ and the bandwidth is narrow. However, it should be noted that in these measurements, the value of the power take-off damping is constant over the whole period range so no optimisation has been carried out on the damping values.

\subsubsection{Results interpretations}

The main discrepancy between experimental data and numerical predictions that is consistently found in the surge and heave responses but also in the piston motion is the difference in wave period for which the peak (or the plunge for the surge response) occurs. This difference is of around 0.06s.

The body mass properties input to the numerical model represent the experimental device with only a limited accuracy. These are obtained by creating a $3 \mathrm{D}$ representation of the device using the 3D mechanical design package SolidWorks. The density of the different materials used for the parts is incor- 

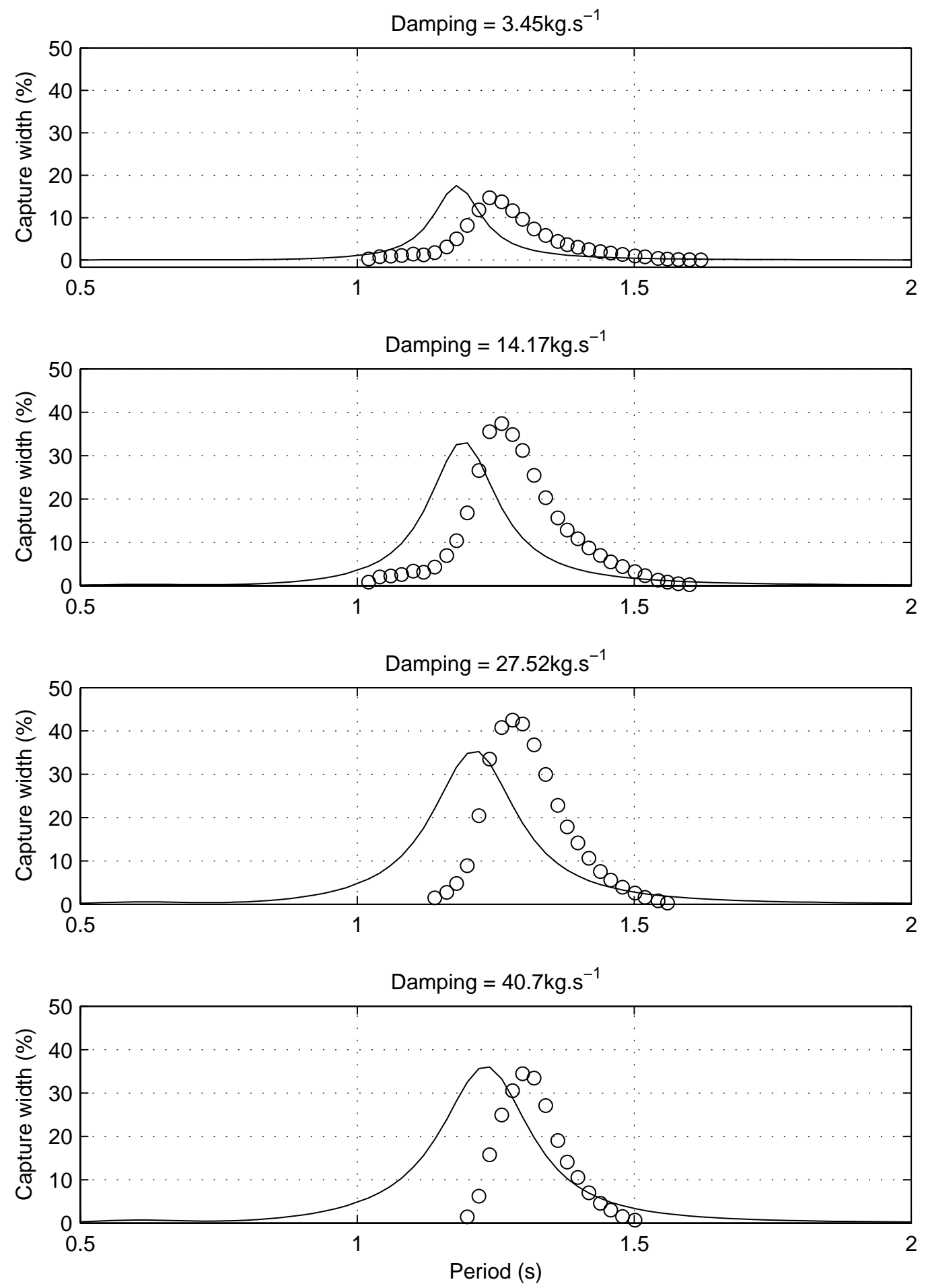

Fig. 15. Comparison between numerical predictions (solid lines) and experimental data (circles) for the capture width of the device. For each case, the damping value applied to the piston motion is indicated above the graph. 
porated in this representation. Due to the complexity of the system, especially the power take off mechanism, details such as screws are generally not taken into account. This leads to an overall discrepancy of $6 \%$ between the measured mass of the device and the mass calculated by SolidWorks.

The mass is one aspect of the 'mass properties'. Moments of inertia and position of the centre of gravity are also very important and difficult to measure accurately. It was thus not possible to check their values calculated from the solid model against actual measurements. Instead, sensitivity analyses for these parameters were carried out on the numerical model to assess their impact on the device's predicted response.

The overall density of the SolidWorks representation was increased in a uniform way to ensure that the overall calculated mass was equal to the measured one. The response predictions were not affected significantly.

The position of the centre of gravity was shifted vertically by up to $\pm 86 \mathrm{~mm}$ without, again, significant impact.

The impact of the moments of inertia was also investigated. Since the motion is planar, only the moment of inertia in pitch is relevant. The moment of inertia $J$ is decomposed into the mass $m$ of the system and the radius of gyration $r$ as follows:

$$
J=m r^{2}
$$

Figure 16 shows the influence of $r$ on the numerical predictions. The five numerical curves are obtained by setting the radius of gyration to respectively $50 \%, 75 \%, 100 \%, 125 \%$ and $150 \%$ of its original value. 

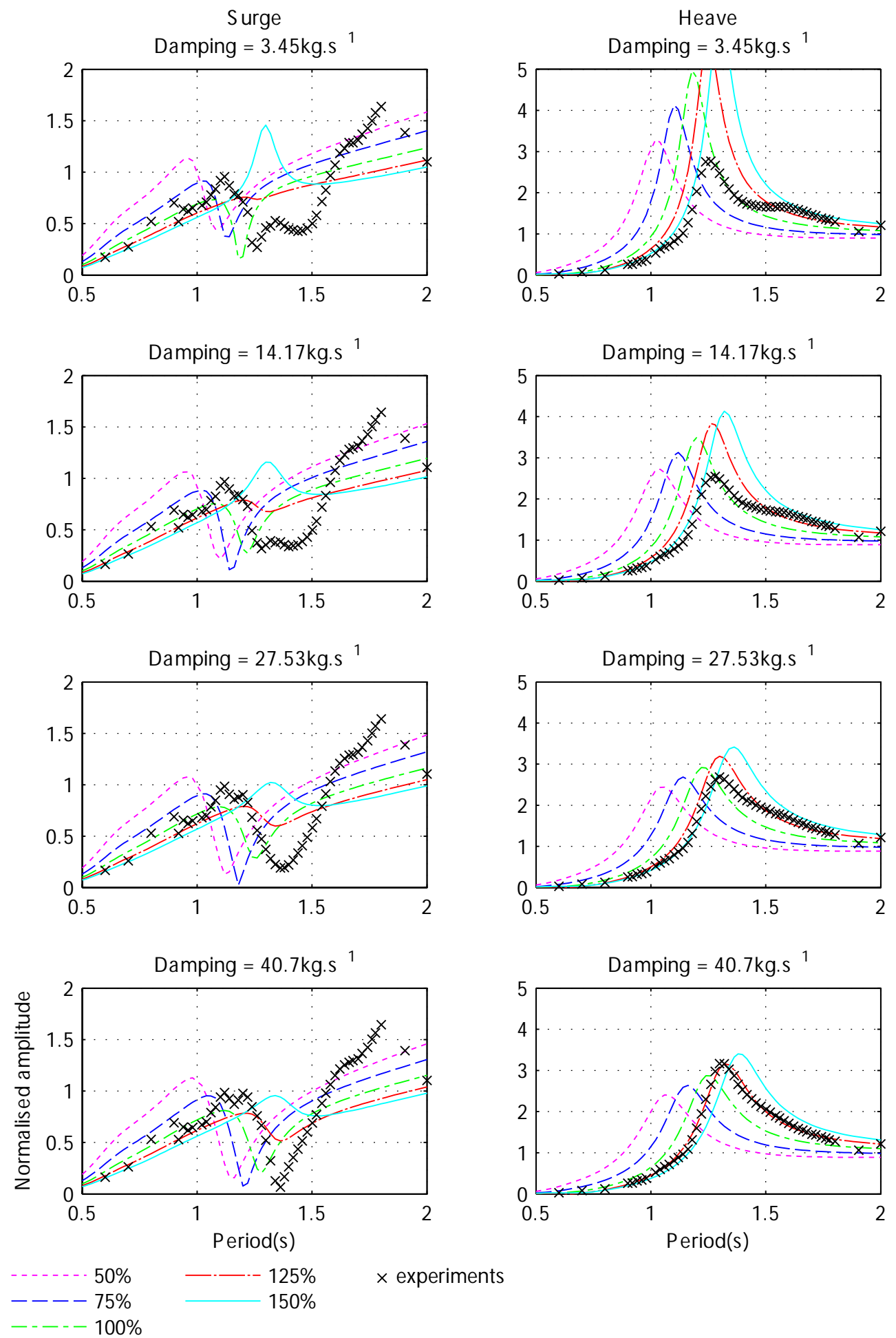

Fig. 16. Impact of variations in radii of gyration on numerical predictions of the device response in surge and heave. The lines correspond to different values of the radius of gyration as a percentage of the original one. The layout of this figure is similar to that of figure 13 . 
It can be seen that the numerical predictions corresponding to radius of gyration $125 \%$ that of the original display a good match with experimental data in terms of wave periods for which peaks (or plunges) occur. Similar observations can be made for the piston response as shown on figure 17 .

A variation in radius of gyration by $25 \%$ corresponds to about $160 \mathrm{~mm}$. This is quite significant and it is arguable whether or not this discrepancy can be fully attributed to inaccuracies of the SolidWorks mass model. This sensitivity analysis however tends to suggest that the difference in wave period between experimental data and numerical predictions is related to mass properties.

\section{Conclusions}

The ability of the boundary-element method to model accurately the configurations of sloped wave energy devices presented in this work is a complex issue. For the configuration without power take-off, numerical modelling was found to be capable of computing the general trend of the device response but was less good at predicting accurate values for the heave amplitude at resonance. Neither did it predict the roll instability. The investigation of the configuration with power take-off proved that it was possible, from a numerical point of view, to model a power take-off mechanism consisting of a piston sliding inside a flooded tube. The comparison with experimental data showed discrepancies in the resonance period which are not fully understood. These results suggest that the validity of linear boundary-element method for this kind of wave energy converter is case specific. Nevertheless, this type of numerical modelling provides useful information on the performance of the devices. In the case of the configuration with power take-off, there are discrepancies between nu- 

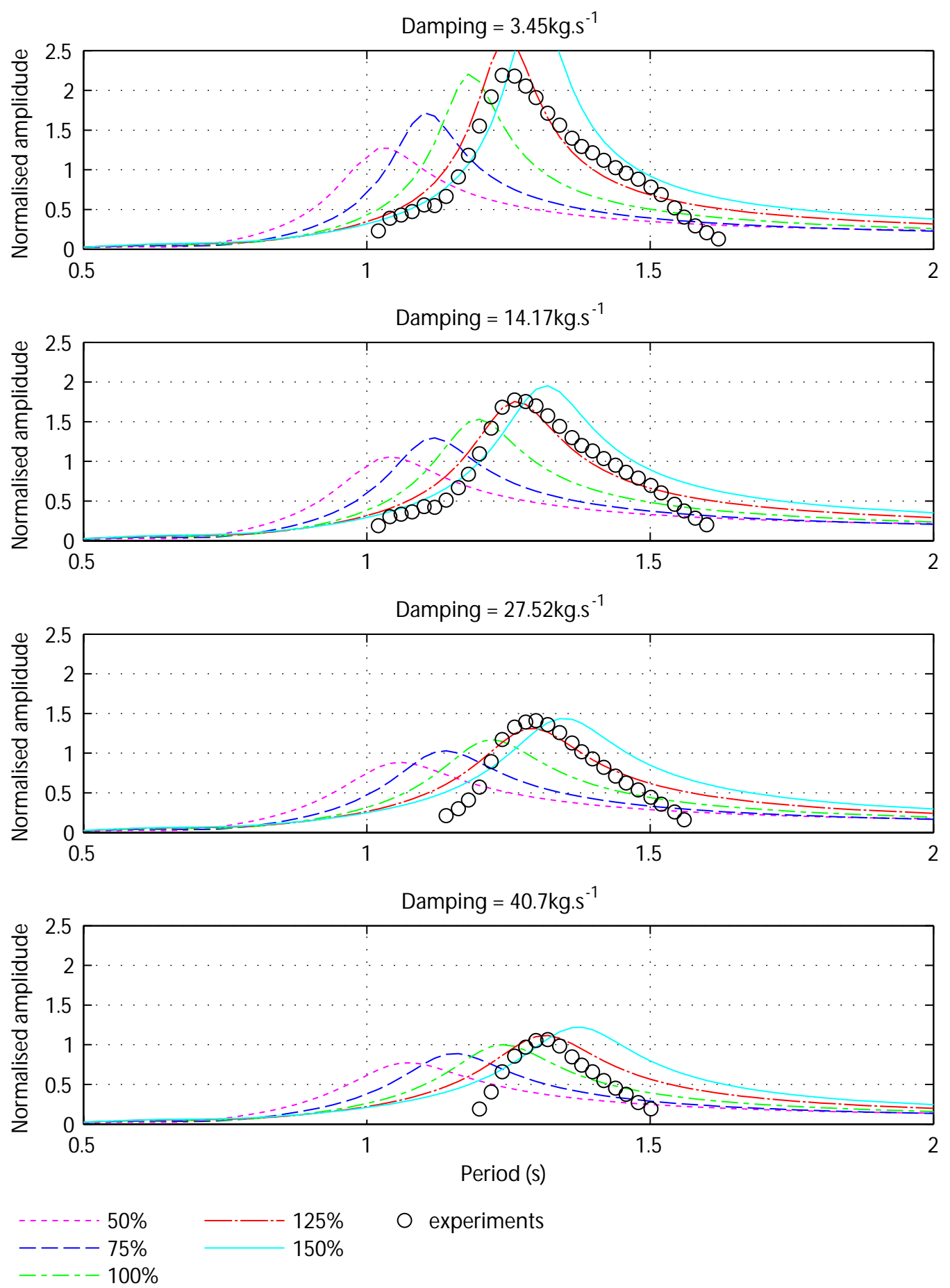

Fig. 17. Impact of variations in radii of gyration on numerical predictions of the piston motion. The lines correspond to different values of the radius of gyration as a percentage of the original one. The layout of this figure is similar to that of figure 14 . 
merical computations and experimental measurements of the capture width. The agreement is good enough however to provide a first assessment of the performance of this configuration as a wave energy converter.

\section{References}

Cruz, J., Pascal, R., Taylor, J. R., June 2006. The Wave Porfile in the Edinburgh Curved Tank. In: 25 ${ }^{\text {th }}$ International Conference on Offshore Mechanics and Arctic Engineering. Hamburg, Germany.

Falnes, J., 2002. Ocean waves and oscillating systems. Cambridge University Press.

Froude, W., 1955. Remarks on Mr. Scott-Russel's Paper on Rolling. In: The Papers of William Froude. Institution of Naval Architects.

Liaw, C., June 1994. Dynamic Instability of a Parametric Excited Ship Rolling Model. International Journal of Offshore and Polar Engineering 4 (2), 105111.

Mollison, D., Buneman, O., Salter, S., 1976. Wave power availability in the NE Atlantic. Nature 263 (5574), 223-226.

Newman, J., 1977. Marine Hydrodynamics. The MIT Press.

Salter, S., Lin, C.-P., November 1995. The sloped IPS wave energy converter. In: $2^{\text {nd }}$ European Wave Energy Conference. Lisbon, Portugal, pp. 337-344. Stansfield, F., 1970. Hydrostatic Bearings. The Machinery Publishing Company Ltd.

Taylor, J., Rea, M., Rogers, D., September 2003. The Edinburgh curved tank. In: $5^{\text {th }}$ European Wave Energy Conference. Cork, Ireland, pp. 307-314.

WAMIT, Inc., 2000. WAMIT user manual Version 6.0, 6.0PC, 5.3S. Available from www.wamit.com. 